\title{
High-Speed Chromatic Dispersion Monitoring of a Two-Channel WDM System using a single TPA Microcavity
}

\author{
K. Bondarczuk ${ }^{1}$, P.J.Maguire ${ }^{1}$, L.P.Barry ${ }^{1}$, J.O'Dowd ${ }^{2}$, W.H.Guo ${ }^{2}$, M.Lynch ${ }^{2}$, A.L.Bradley ${ }^{2}$, J.F.Donegan ${ }^{2}$ \\ (1) Research Institute for Networks and Communications Engineering, Dublin City University, Glasnevin, Dublin 9, Ireland \\ (2)Semiconductor Optronics Group, University of Dublin, Trinity College, Dublin 2, IRELAND \\ e-mail: krzysztof@eeng.dcu.ie
}

\begin{abstract}
Chromatic dispersion monitoring of two $160 \mathrm{~Gb} / \mathrm{s}$ wavelength channels using a TPA Microcavity is presented. As the microcavity exhibits a wavelength resonance characteristic, a single device could monitor a number of different WDM-channels sequentially. (C)2008 Optical Society of America

OCIS codes: (320.7110) Ultrafast nonlinear optics, (060.2330) Fiber optics communications
\end{abstract}

\section{Introduction}

Due to continued growth of the Internet and the introduction of new broadband services, it is expected that individual channel line rates will exceed $100 \mathrm{~Gb} / \mathrm{s}$ in the next 5 years. One of the major limitations to operating at such high data rates will be the amount of Chromatic Dispersion (CD) introduced. Dispersion management can be used, but it will not compensate for additional CD fluctuations that arise due to variation of temperature [1], or mechanical stress. Such variations will become a serious problem when individual line rates exceed $40 \mathrm{~Gb} / \mathrm{s}$. It is important to develop new CD monitoring techniques that have the ability to operate at such high data rates. In this paper a technique based on the optical nonlinearity of Two-Photon Absorption (TPA) in a specially designed microcavity is utilized for wavelength selective, CD monitoring of two WDM channels, each operating at 160Gb/s. TPA is a nonlinear optical-to-electrical conversion process that occurs in semiconductors when two photons are simultaneously absorbed to generate a single electron-hole pair. The TPA photocurrent is proportional to the square of incidence optical power falling on the detector. It is this nonlinear response that allows TPA to be used for CD monitoring. The average TPA photocurrent generated by an incident optical signal with a power envelope $P(t)$ is given by [2]:

$$
I_{T P A}^{A v} \propto \frac{P_{a v e}^{2}}{D} \quad \text { where } \quad D=\frac{\langle P(t)\rangle^{2}}{\left\langle P^{2}(t)\right\rangle}
$$

where $D$ is the generalized duty cycle and $\langle\ldots>$ means time averaging. As the duty cycle increases with $\mathrm{CD}$, the average TPA photocurrent will change accordingly, allowing for the CD monitoring of ultra-fast optical signals without the need for fast electronics [3]. One way to overcome the inherent inefficiency associated with TPA is to employ Resonance Cavity Enhancement (RCE) technology within the detector. One interesting feature of using a RCE-based device is the fact that the incident signal is only enhanced over a narrow wavelength range determined by the device design [4]. This allows the monitoring of a single wavelength channel without the necessity of using an additional external optical filter. Also as the cavity resonance wavelength peak can be easily tuned by tilting the device, allowing different wavelength channels to be monitored using a single device.

\section{Experimental results}

Before carrying out the experimental work, the TPA microcavity used was initially characterized. Fig.1(a) displays the cavity resonance characteristic for the device, with a measured $3 \mathrm{~dB}$ cavity bandwidth of $265 \mathrm{GHz}(2.1 \mathrm{~nm})$. Fig.1(b) shows that the resonance wavelength tunability of the microcavity exceeds $80 \mathrm{~nm}$, allowing the entire C-band to be monitored using a single device. The experimental setup for TPA-based CD monitoring is shown in Fig.1(c). Two $10 \mathrm{GHz}$ RZ optical pulse trains were generated using wavelength tunable (1480-1580nm) actively mode-locked pulse sources. The wavelength channels were separated spectrally by $10 \mathrm{~nm}$, operating at wavelengths of $1556 \mathrm{~nm}$ and $1546 \mathrm{~nm}$ respectively. Both channels were combined and modulated with $2^{7}-1$ RZ PRBS data signal. Each channel then entered a $10-160 \mathrm{~Gb} / \mathrm{s}$ bit rate multiplexer, consisting of four independently switch-able stages. The higher bit rate WDM signal then passed through various lengths $(0-80 \mathrm{~m})$ of dispersion compensating fiber ( $\mathrm{D}=-99.3 \mathrm{ps} / \mathrm{km} . \mathrm{nm}$ ) before being incident on the TPA microcavity. The power level falling on the TPA microcavity was fixed at $6 \mathrm{dBm}$. The generated photocurrent for the varied DCF lengths and multiplexed data rates were measured on the PicoAmmeter. Initially the cavity was set to normal incidence angle to monitor the CD of the first 


\section{CThAA7.pdf}

channel $\left(1556 \mathrm{~nm}\right.$ ). The cavity was then tilted by $22^{\circ}$ (cavity resonance wavelength shifted by $10 \mathrm{~nm}$ ) to monitor the second channel at $1546 \mathrm{~nm}$.
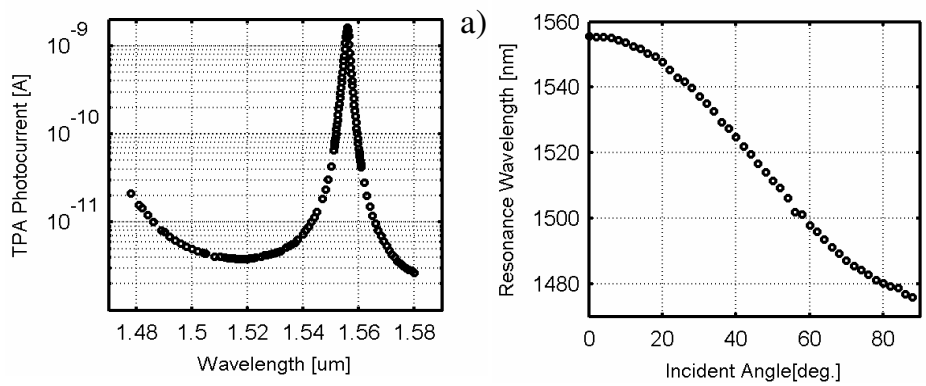

b)

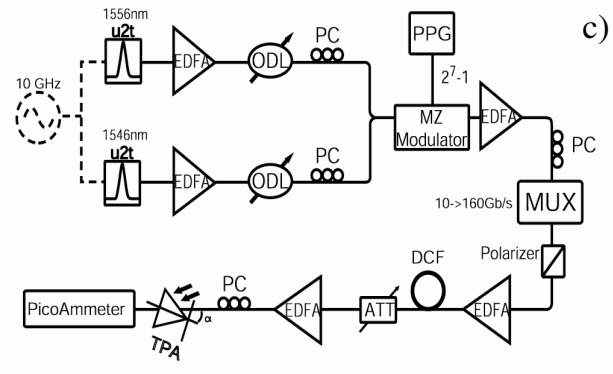

Fig. 1 (a) Cavity resonance characteristic, (b) Cavity resonance wavelength for varied incident angle, (c) Experimental Setup

The experimental results are shown in Fig.2 (a) for the first channel and in Fig.2 (b) for the second channel. The TPA photocurrent decreases with an increase in the accumulated dispersion (ps/nm) in accordance with Eq.1. Both signals flatten out at the same level for given incident angle. The levels however, differ for tilted device since the cavity enhancement factor depends on the incident angle. Therefore the optical field within cavity and the generated TPA photocurrent decreases with increased angle. As can be seen for both wavelength channels the monitoring range is limited by pulse overlapping to $4 \mathrm{ps} / \mathrm{nm}$ for $160 \mathrm{~Gb} / \mathrm{s}$ and $6 \mathrm{ps} / \mathrm{nm}$ for $80 \mathrm{~Gb} / \mathrm{s}$. Above these threshold values the overlapped pulses can combine coherently resulting in reconstruction of the original periodic signal (Talbot effect [5]). In our experiment however, this self-imaging effect is suppressed by the PRBS modulation and by the fact that coherency of signal degrades after propagation through a system. The residual reconstruction can be visible for $160 \mathrm{~Gb} / \mathrm{s}$ signal above the $4 \mathrm{ps} / \mathrm{nm}$ threshold. The overall effect of this pulse (coherent or incoherent) overlapping will be to limit the range over which the $\mathrm{CD}$ of the signal can be monitored. As a result, this techniques is more suited for monitoring of small levels of CD fluctuations in high-speed ( $>100 \mathrm{~Gb} / \mathrm{s})$ optical communications systems.

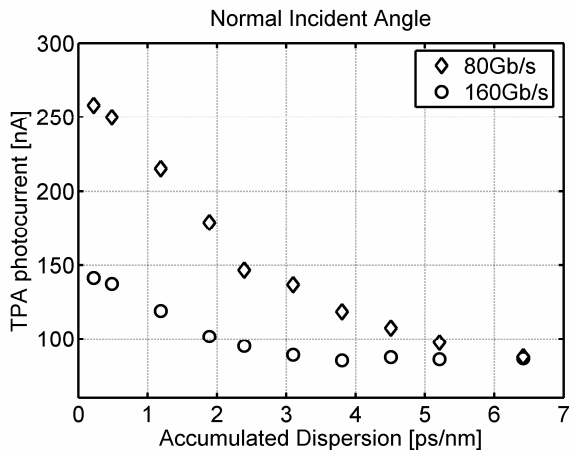

a)

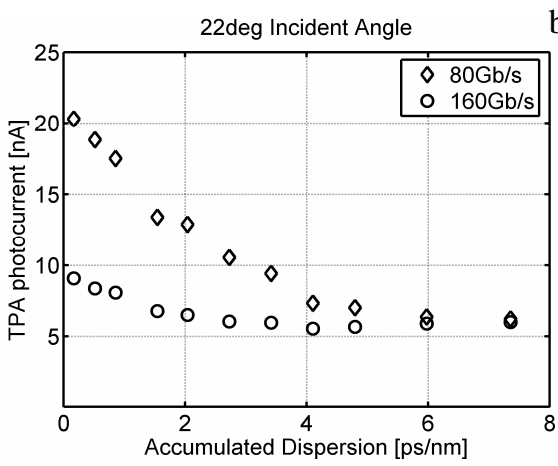

b)

Fig. 2. CD monitoring of $80 \mathrm{~Gb} / \mathrm{s}$ and $160 \mathrm{~Gb} / \mathrm{s}$ data signal at (a) $1556 \mathrm{~nm}$ (normal incident angle) and (b) $1546 \mathrm{~nm}\left(22^{\circ}\right.$ incident angle)

\section{Conclusion}

The experimental results demonstrate CD monitoring of two, high-speed WDM signals using a single TPA microcavity. Due to the ultra-fast nature of the TPA effect, and the resonance response of the microcavity, a single device can be employed to monitor a large number of WDM channels, each operating at a data rate exceeding $100 \mathrm{~Gb} / \mathrm{s}$. Such a device is ideally suited for the monitoring of small fluctuations of CD arising from variation of temperature or mechanical stress.

\section{References}

[1] Takatoshi Kato, Yasushi Koyano and Masayuki Nishimura, "Temperature Dependence of Chromatic Dispersion in Various Types of Optical Fibers," OFC 2000, 1, pp. 104-106, paper TuG7.

[2] Stephan Wielandy, Michael Fishteyn, Benyuan Zhu, “Optical Performance Monitoring Using Nonlinear Detection,” J.Lightwave Technol, 22, no. 3, pp: 784-793 (2004)

[3] K. Bondarczuk, P. J. Maguire, L. P. Barry, J. O'Dowd, W.H. Guo, M. Lynch, A.L. Bradley, J.F. Donegan, H. Folliot, "Chromatic Dispersion Monitoring of 80-Gb/s OTDM Data Signal via Two-Photon Absorption in a Semiconductor Microcavity," PTL, 19, no. 1, pp.21-23 (2007)

[4] H.Folliot, M.Lynch, A.L.Bradley, L.A.Dunbar, J.Hegarty, J.F.Donegan, L.P.Barry, J.S.Roberts, G.Hill, "Two-photon absorption photocurrent enhancement in bulk AlGaAs semiconductor microcavities," Appl. Phys. Lett, 80, no.8, pp.1328-1330, (2002).

[5] Dominik Pudo, Michal Depa, Lawrence R. Chen, "Single and Multiwavelength All-Optical Clock Recovery in Single-Mode Fiber Using the Temporal Talbot Effect," J.Lightwave Technol, 25, no. 10, (2007) 